\title{
Perinatal Service in the State of Qatar
}

\section{Sajjad Ur Rahman ${ }^{1}$ and Badreldeen Ahmed ${ }^{2,3 *}$}

${ }^{1}$ Women's Hospital Hamad Medical Corporation, Doha, Qatar

${ }^{2}$ Clinical Pediatrics, Feto Maternal Centre, Doha, Qatar

${ }^{3}$ Fetal Medicine Centre, Weill Cornell Medical College, Qatar

\begin{abstract}
Maternal mortality rate (MMR): Qatar's MMR dropped from 49/100,000 in 1990 to $9.02 \%$ in 2012. Qatar's MMR had been zero for several years between 1995 and 2000; for the rest of the years, it had been between 7 and 11/100,000. Qatar's MMR over the last decade has not only been significantly low, as compared to the global MMR of 260/100,000 and regional MMR of 320/100,000 in Eastern Mediterranean Region as given, it is also comparable to the MMR of many high income countries, both from the West and East. Qatar's antenatal coverage is $94 \%$. Qatar's reproductive health system is based on $99.45 \%$ hospital deliveries. During 2011, the State of Qatar had a $25 \%$ national C section rate. The relative risk of neonatal mortality was significantly higher among $\mathrm{C}$ section deliveries, particularly in emergency $\mathrm{C}$ Section deliveries.
\end{abstract}

Neonatal mortality and perinatal mortality rates: The Relative Risk of Neonatal Mortality in Qatar decreased by $87 \%$ between 1975 and 2011, though the population increased by 10 fold and number of deliveries by 7.2 folds. This is a superb achievement; which encompasses both early and late neonatal mortality. Qatar's neonatal mortality rate corrected for lethal congenital anomalies and futility (cNMR) was 3.26/1000 during 2011. Qatar's 2011 NMR (4.9/1000) and cNMR (3.26/1000) are comparable to selected high income countries; both from the west and east.

Keywords: Perinatal medicine; Qatar; Maternal mortalty; Neonatal mortality; Progress

\section{Introduction}

State of Qatar is a sovereign country in the Middle East. Geographically, a peninsula spread over $11,437 \mathrm{Sq} \mathrm{km}$; it is bordered by Saudi Arabia in the South and Persian Gulf on all other sides. Currently, Qatar is the world's largest Liquefied Natural Gas (LNG) producer [1]. Since 2010, Qatar has the highest per capita GDP in the world, which is based on its proven reserves of oil and natural gas [2]. Qatar's economic boom, which started in mid-1990, has not only resulted in exponential increase in its population due to economic migration; it has also resulted in a parallel increase in the number of deliveries and births, approaching 20,000 deliveries per year. Qatar's current population (1.8 million) is comprised of 30\% native Qataris and 70\% expatriates (Arab nations 20\%; India 20\%, Nepal 13\%, Philippines 10\%, Pakistan 7\%, Sri Lanka 5\% and other countries 5\%) [2,3]. Life in Qatar is changing very fast due to heavy investment in infrastructure and systems development. This includes establishing an equitable health system, with universal access to health care for all citizens [4]. This is evident from the fact that the State of Qatar allocated 27\% of its national budget for health care during 2011 [4]. United Nations has classified Qatar as one of the 42 countries possessing a very high human development index, which is evident from the fact that The State of Qatar is heavily investing in research, including health related research, through Qatar National Research Fund (QNRF) [5].

Estimating and reporting reproductive outcomes, their correlates and risk factors at a national level is a daunting task. For the State of Qatar, Pearl Study (Perinatal Neonatal Outcomes Research Study in the Arabian Gulf) has now achieved this for maternal, neonatal and perinatal mortality outcomes during 2011 and their trends, since 1975. According to the PEARL Study, Qatar's 2011 maternal, neonatal and perinatal outcomes are comparable to many high income countries, both from the West and East.

Qatar had only two maternal deaths during 2011, giving a Maternal Mortality Rate (MMR) of 9.85/100,000. Qatar's MMR had been zero for several years between 1995 and 2000; for the rest of the years, it had been between 7 and 11 (Figure 1). This is a great achievement against a global MMR of 260/100,000 and regional MMR of 320/100,000 in the Eastern Mediterranean Region (EMR). Qatar MMR is comparable to many high income countries, both from the West and East (Figure 2) [6].

\section{Improvement of Maternal Mortality (MDG-5-A)}

During 2011, a total of 491 women died in Qatar, out of which 172 were in the reproductive age group (15-55 years). Both maternal deaths mentioned before occurred in public sector tertiary maternity hospital. One of these two deaths occurred in the medical ICU, secondary to HELPP Syndrome and its complications (fulminate hepatic failure, abdominal bleeding and multiple organ failure). The second death occurred on the maternity unit due to sudden pulmonary embolism.

During 2012, a total of 446 women died in Qatar, out of which 158 were in the reproductive age group (15-55 years). During 2012, Qatar had three early maternal deaths. One of these was due to sudden cardiac arrest, 33 days post-delivery in a woman who was previously completely asymptomatic. One of these had septic abortion during first trimester, and the other one had military tuberculosis and septic shock with multi organ failure at 18 weeks of gestation.

Between 1990 and 2012, Qatar's MMR dropped by 80\% (from 49/100,000 in 1990 to $9.02 \%$ in 2012). Qatar's MMR had been zero for several years between 1995 and 2000; for the rest of the years, it had

*Corresponding author: Badreldeen Ahmed, Associate Professor, Clinica Pediatrics, Fetal Medicine Centre Doha, PO Box 3050 Doha, The State of Qatar Tel: 00974 44756369; Fax: 0097444756367; E-mail: profbadreldeen@hotmail.com

Received April 25, 2013; Accepted June 12, 2013; Published June 19, 2013

Citation: Rahman SU, Ahmed B (2013) Perinatal Service in the State of Qatar. J Health Med Informat S11: 012. doi:10.4172/2157-7420.S11-012

Copyright: (c) 2013 Rahman SU, et al. This is an open-access article distributed under the terms of the Creative Commons Attribution License, which permits unrestricted use, distribution, and reproduction in any medium, provided the original author and source are credited. 


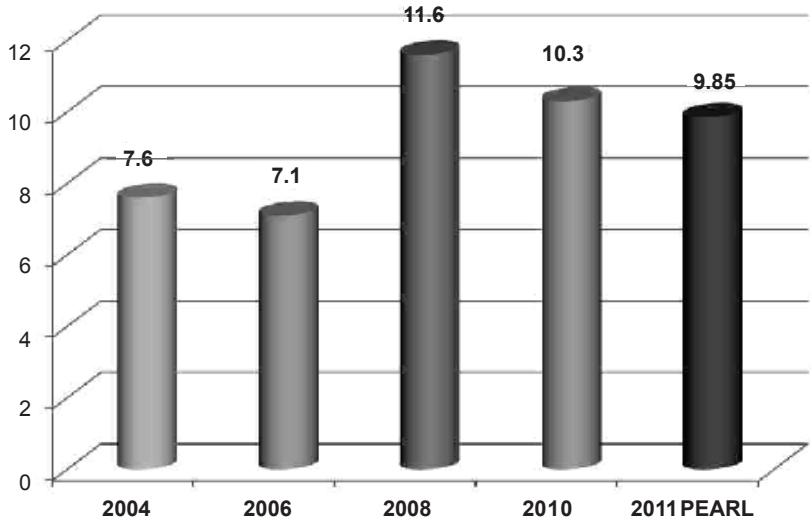

Figure 1: Trends in maternal mortality rates (per 100,000) in Qatar (20042011).

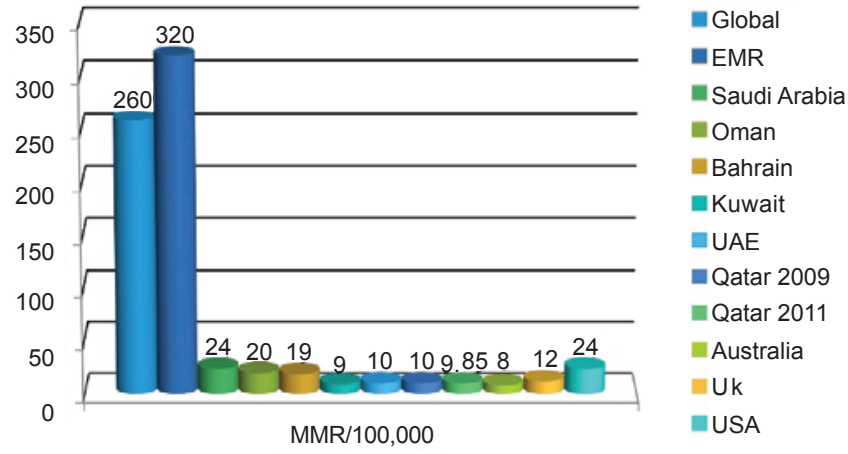

Figure 2: Qatar's MMR (per 100,000), as estimated by PEARL Study during 2011, compared with Global, EMR, GCC Countries and selected high income countries. Source: World Health Statistics 2011.

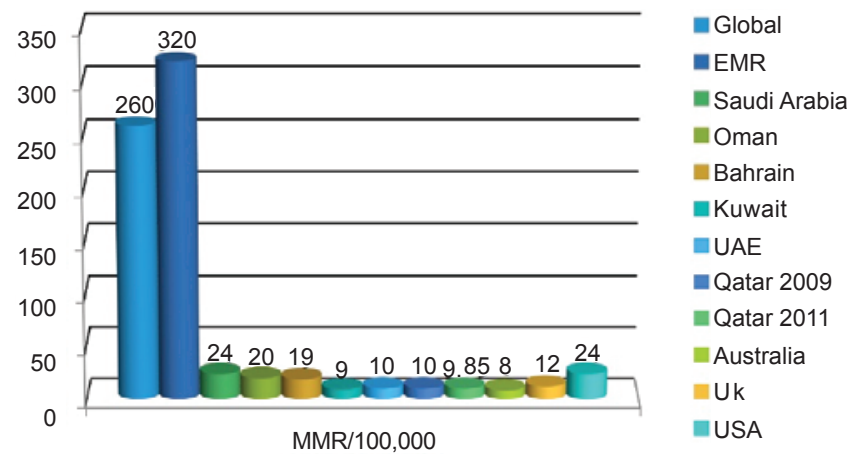

Figure 3: Qatar's MMR (per 100,000), as estimated by PEARL Study during 2011, compared with Global, EMR, GCC Countries and selected high income countries. Source: World Health Statistics 2011.

been between 7 and 11/100,000 (Figure 1). Qatar's MMR over the last decade has not only been significantly low as compared to the global MMR of 260/100,000 and regional MMR of 320/100,000 in Eastern Mediterranean Region (EMR), as given in the WHO's World Health Statistics 2011; it is also comparable to the MMR of many high income countries, both from the West and East (Figure 2).

Qatar presently enjoys an outstanding antenatal coverage of $94 \%$.

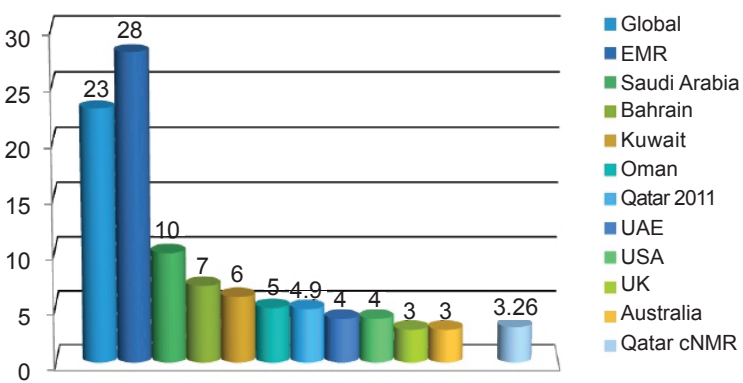

Figure 4: Qatar's NMR (per 1000) during 2011 compared with Global, EMR, GCC and few developed high income countries' NMRS. Sourece: World Health Statistics 2011.

Qatar's reproductive health system, based on $99.45 \%$ hospital based deliveries. During 2011, the State of Qatar had a 25\% national C section rate. According to PEARL Study analysis, the Relative Risk of Neonatal Mortality was significantly higher among $C$ section deliveries ( $p=0.003)$, particularly Emergency C Section deliveries $(p<0.001)$.

\section{Improvements of Neonatal Mortality and Perinatal Mortality}

Between 1975 and 2011, the Relative Risk of Neonatal Mortality in Qatar decreased by $87 \%(p<0.001)$ though the population increased by 10 fold, and number of deliveries by 7.2 folds. This is a superb achievement; which encompasses both Early and Late Neonatal Mortality (Figure 3). Qatar's Neonatal Mortality rate corrected for lethal congenital anomalies and futility (cNMR) during 2011 was 3.26/1000. Qatar's 2011 NMR (4.9/1000) and cNMR (3.26/1000) are comparable to selected high income countries, both from the West and East (Figure 4).

\section{Summary}

Perinatal service in Qatar is witnessing continuous changes. The maternal and neonatal service is of highest quality and continues to grow. The number of deliveries is increasing at a faster pace, compared to western societies. There is now a great emphasis on research on area of perinatal medicine, and the Pearl study have provided us with great insight to the standard of the perinatal care in Qatar.

Qatar's MMR for 2011 and 2012 is one of the lowest among the regional countries and comparable with the MMR of selected high income countries. In addition, Qatar has achieved both components of Millennium Development Goal-5, before the target year of 2015 . However, this achievement should act as a catalyst in developing strategies for further improvement in reproductive, maternal and child health care.

\section{Acknowledgment}

This paper is based on the executive synopsis annual report of the Pearl Study (Perinatal Neonatal Outcomes Research Study in the Arabian Gulf).

\section{References}

1. WHO (2013) World Blood Donor Day: More voluntary blood donors needed WHO, France.

2. Qatar.

3. (2013) Qatar Statistics Authority.

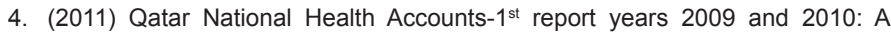
baseline analysis of health expenditure and utilization.

5. (2013) Qatar National Research Fund

6. WHO (2011) World Health Statistics 2011. WHO, France 